\title{
Management of a Dentigerous Cyst in a Child with Robin Sequence
}

\author{
Shayda Mirhaidari ${ }^{1,2}$, Ananth Murthy ${ }^{2,3}$ \\ ${ }^{1}$ Division of Plastic and Reconstructive Surgery, Summa Health System, Akron, $\mathrm{OH} ;{ }^{2}$ Northeastern Ohio Medical University, Rootstown, $\mathrm{OH}$; \\ ${ }^{3}$ Akron Children's Hospital, Akron, OH, USA
}

This is a brief clinical report describing an 18-month-old female with Robin sequence found to have an incidental mandibular cystic lesion on a head computed tomography scan in the preoperative workup before performing mandibular distraction. She underwent enucleation of the tumor, which was found to be a dentigerous cyst. One year following cyst enucleation, mandibular distraction was performed in order to alleviate her tongue-based obstruction. This case demonstrates the ability of the mandibular bone to successfully regenerate after undergoing cyst enucleation.

Keywords Dentigerous cyst / Pierre Robin sequence / Mandible / Enucleation
Correspondence: Ananth Murthy Division of Plastic Surgery, Akron Children's Hospital, 215 West Bowery St., Suite 3300 , USA

Akron, Ohio 44308, USA

Tel: +1-330-543-4970

Fax: +1-330-543-4971

E-mail: amurthy@chmca.org

The authors have no potential conflicts of interest in relation to the content of this article.

Received: 24 Apr 2016• Revised: 21 Jan $2017 \bullet$ Accepted: 22 Jan 2017

pISSN: 2234-6163 • elSSN: 2234-6171 • https://doi.org/10.5999/aps.2017.44.5.434• Arch Plast Surg 2017;44:434-438

\section{INTRODUCTION}

Dentigerous cysts (DCs) are the second most common type of odontogenic (developmental) cyst arising in the jaw [1]. They are defined as cystic lesions that develop around the crown of an unerupted tooth, in which follicle expansion allows for fluid accumulation. Also known as follicular cysts, DCs account for approximately $24 \%$ of true jaw cysts [2].

The most commonly affected teeth are the mandibular third molars and the maxillary canines, which may be explained by the tendency of these teeth to present with impaction [3]. DCs have a slight male predominance, and are most likely to occur during the second and third decades of life [4]. DCs diagnosed during childhood are rare, with most cases in the literature described in small series or case reports [4]. DCs are usually solitary in nature. When presenting as bilateral or multiple cysts, they are usually associated with syndromes such as mucopolysaccharidosis, basal cell nevus syndrome, or cleidocranial dys- plasia [5].

Another uncommon jaw anomaly found in infancy is Robin sequence (RS). RS is characterized by micrognathia, cleft palate, and glossoptosis. The treatment of this condition frequently utilizes distraction osteogenesis to correct micrognathia. We present a brief clinical report of an incidental dentigerous cyst in a child with RS and its surgical management.

\section{CASE}

An 18-month-old female previously had a tracheostomy placed in infancy to treat an airway obstruction secondary to RS. The recommendation was to first correct the cleft palate, followed by mandibular distraction in order to decannulate the child.

The cleft palate was closed at 19 months of age. A 2-flap palatoplasty with levator retropositioning was performed. The patient's postoperative course was uneventful. Two months following cleft palate closure, the patient was re-evaluated for man- 
dibular distraction. A preoperative computed tomography (CT) scan demonstrated an incidental cystic lesion of the left mandibular ramus measuring $3.9 \mathrm{~cm}$ in length and $2.2 \mathrm{~cm}$ in the transverse dimension with a floating molar tooth. The mass was unilocular and distorted both cortices of the mandible. It appeared to be emanating from the second molar on the left side and extended to the level of the condyle. In order to obtain a definitive diagnosis, the decision was made to enucleate the mass.

At the age of 25 months, the patient underwent enucleation of the mandibular cyst. A subperiosteal exposure was performed. Upon making the osteotomy, copious amounts of yellow-colored, almost purulent fluid were expressed. The mass had obliterated both cortices of the bone. The floating teeth and surrounding bone were removed and curetted. The periosteum was intact and the only portion of viable bone was along the posterior border of the ramus. No bone grafting was performed (Figs. 1A, 2A). The intraoral incision healed without any complications and observation was recommended.

Surgical pathology identified the mass as a dentigerous cyst. Multiple sections revealed a cyst composed of dense fibrous tissue lined by squamous epithelium. Ameloblastic epithelium was not identified, and there was no evidence of carcinoma. Culture of the cyst aspirate showed the growth of many beta-hemolytic group F streptococci and possible anaerobic species. Also present were 2 molar-like tooth structures, which measured $1.1 \mathrm{~cm}$ $\times 1.0 \mathrm{~cm} \times 0.6 \mathrm{~cm}$ and $1.1 \mathrm{~cm} \times 0.9 \mathrm{~cm} \times 1.2 \mathrm{~cm}$, with the crown aspect of 1 of them projecting into the lumen of the cyst.

At 3 and a half years of age, the patient continued to demon- strate an inability to tolerate capping of the tracheostomy. An approximately 16-mm overjet was present centrally. The tongue remained posteriorly displaced. A CT scan showed a regenerated mandibular ramus that could accommodate a distraction (Figs. 1B, 2B).

At that time, the patient underwent successful bilateral mandibular osteotomy and distraction. Approximately 2.5 months later, the internal distractors were removed. Two months later, she was decannulated. A follow-up CT scan performed 2 years postoperatively demonstrated bone stock after distraction (Fig. 2C).

\section{DISCUSSION}

The various theories on the histogenesis of dentigerous cysts include developmental and inflammatory origins. The developmental origin is associated with an unerupted tooth exerting pressure on a follicle, impeding venous return, and in turn allowing for cystic fluid accumulation to develop between the reduced enamel epithelium. It has also been suggested that the inflammation of deciduous teeth in nearby proximity to an unerupted tooth can trigger cyst formation. This process is usually seen in the first and second decades of life [6]. Nevertheless, DCs do occur in childhood, although they are uncommon. Only 9.1\% of DCs occur in the 6- to 7-year-old population [7]. The diagnosis of a DC in our patient at 21 months represents one of the few diagnoses to be reported in the literature in such a young patient [8].

\section{Fig. 1. CT scan before and after enucleation}

(A) Computed tomography (CT) scan demonstrating a cystic lesion measuring $3.9 \mathrm{~cm} \times 2.2 \mathrm{~cm}$ in the left mandibular ramus associated with a floating molar tooth. The red arrow points to the cyst (21 months of age). (B) CT scan at the age of 3.5 years demonstrating good bone formation after enucleation.
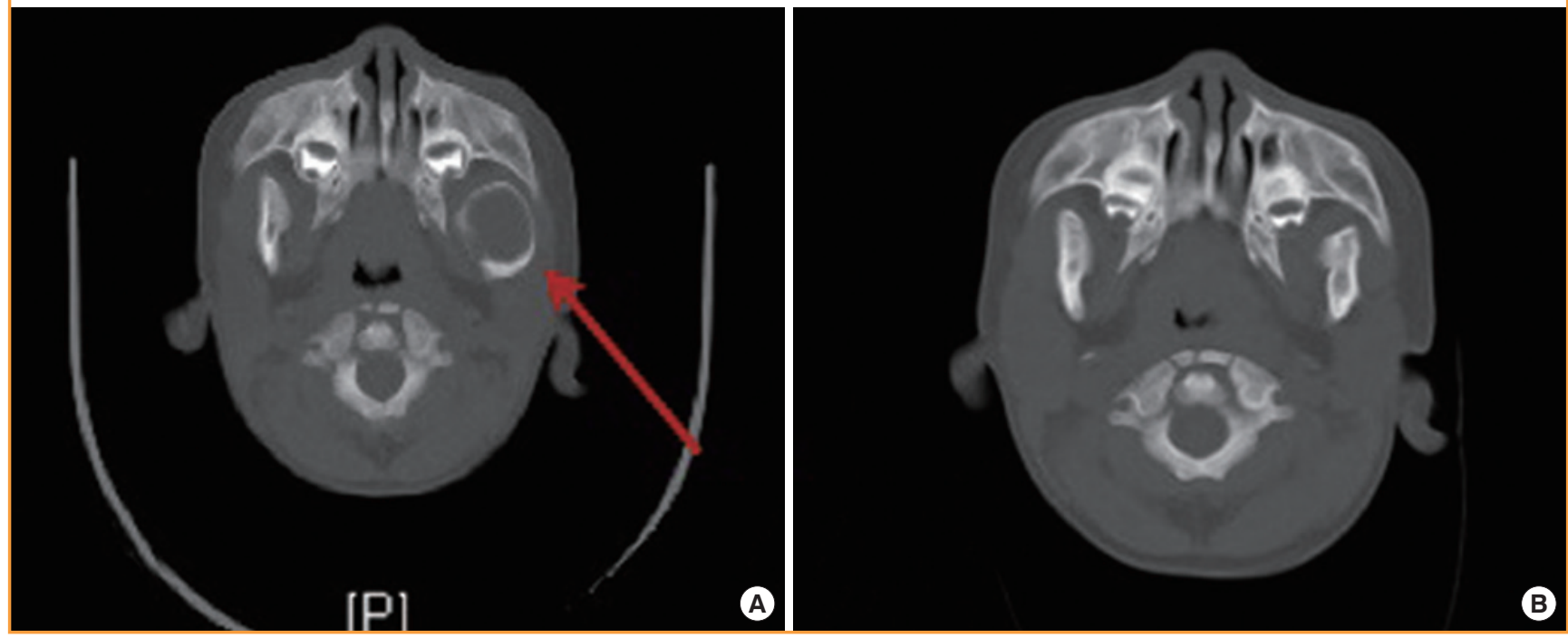


\section{Fig. 2. CT scan before and after mandibular distraction}

(A) Computed tomography (CT) scan demonstrating a large cystic lesion of the left mandibular ramus. The cyst is shown by an red arrow. (B) CT scan at the age of 3.5 years (18 months after enucleation) demonstrating loss of second molar but adequate bone formation. (C) CT scan performed 2 years after bilateral mandibular osteotomy and distraction.
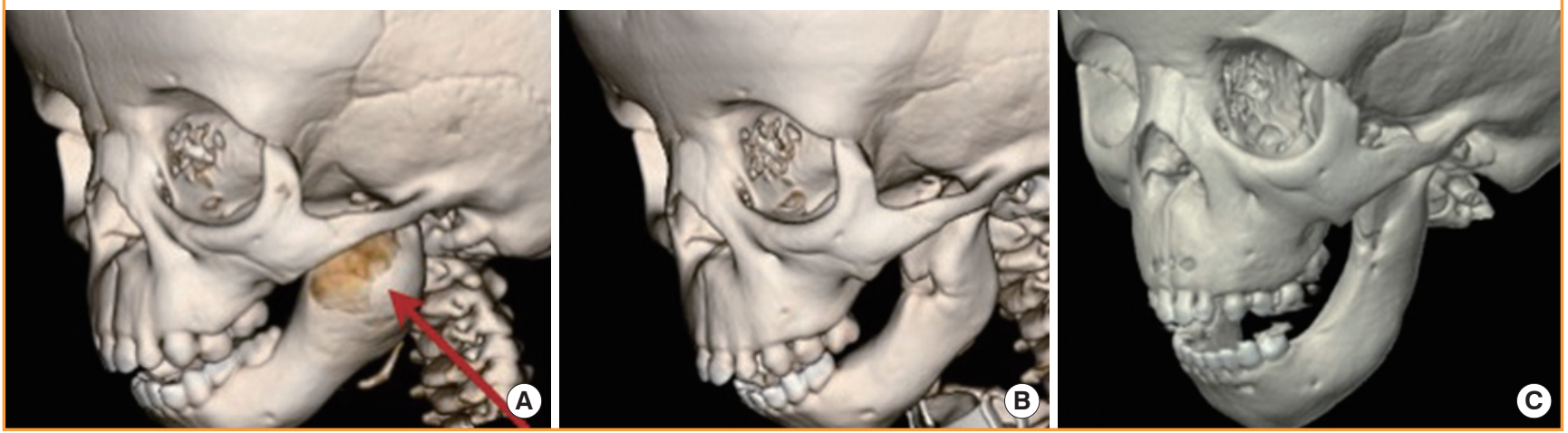

Most commonly, DCs present as asymptomatic mandibular swellings, but they may also present with unilateral jaw pain when they are inflammatory in nature. In this patient, the DC was incidentally found on a preoperative CT scan in anticipation of mandibular distraction. In a retrospective review of prior imaging, evidence of the lesion was identified. An approximately 14-mm unilocular lucency in the left mandible was present on a chest X-ray for tracheostomy placement at 13 months of age (Fig. 3). All other imaging prior to this date did not elucidate the mandible well.

It is difficult to discern the origin of the cyst in this patient. The lower second molars typically erupt at 23-31 months [9]. It is possible that the unerupted tooth in this location exerted pressure on an impacted follicle, leading to fluid accumulation and eventual cyst formation. With evidence of the cyst on imaging at 13 months, a developmental origin could explain its presence. The other possibility would be an inflammatory origin. Our patient had been admitted for multiple upper respiratory tract infections requiring intensive care unit hospitalization. The final pathology report demonstrated the presence of beta-hemolytic group F streptococci, suggesting an underlying infection. It is possible that the underlying inflammation of a non-vital deciduous tooth or infection from another source spread to involve the follicle of an unerupted tooth, resulting in inflammatory debris and eventual cyst formation [6].

Radiographically, DCs appear as a well-defined unilateral radiolucency surrounding the crown of an unerupted tooth. A follicular space of $>4 \mathrm{~mm}$ is suspicious for an underlying cyst. Other odontogenic lesions exist, with similar radiographic findings. The differential diagnosis includes radicular cysts, ameloblastoma, squamous cell carcinoma, chondrosarcoma, osteosarcoma, cementoblastoma, and Pindborg tumor. The complications that can arise from untreated DCs include: pathologic
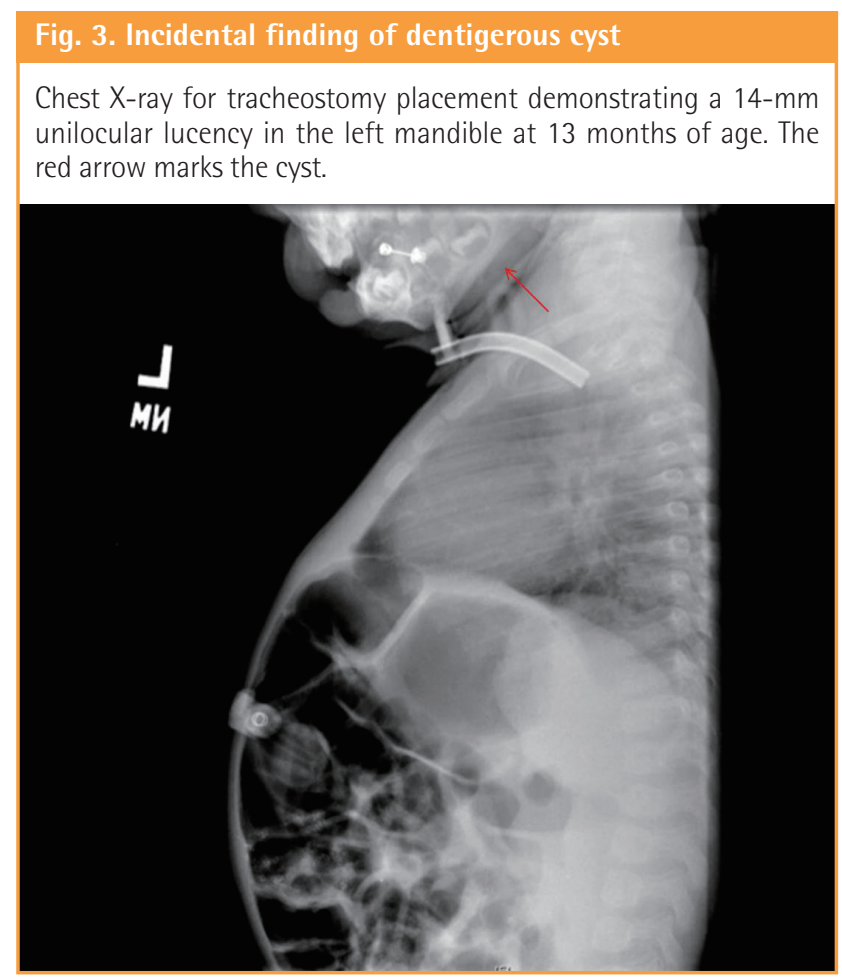

bone fracture/destruction, loss of permanent teeth, and permanent bone deformation, leading to facial asymmetry and the displacement of teeth [10]. Debate currently centers around whether marsupialization or enucleation is the best treatment modality in the management of DCs. Enucleation is the treatment of choice, in which the cyst and all of its contents are removed in toto. This is the preferred method if supernumerary teeth are involved, and if this is the case, the unerupted tooth is also removed. The disadvantages of enucleation include the potential to affect budding teeth, especially in children. Therefore, marsupialization is often the preferred method in children, as the loss of permanent tooth buds can be prevented [4]. This 
more conservative approach involves decompressing the cyst and suturing its lining to the oral mucosa to allow for continuous drainage. The obvious disadvantage of this technique is the potential for cyst recurrence as well as for pathologic tissue to be left in situ [11].

Our patient presented a unique challenge in that the cyst was quite large $(4 \mathrm{~cm})$ and had replaced almost all of the mandibular ramus. It was apparent that the mass had obliterated the bone, on both the lingual and buccal cortices. Fortunately, after the mass was removed, viable bone was present along the posterior edge of the ramus connecting to the body and condyle, and the periosteum was intact.

Spontaneous regeneration of the mandible is an infrequent response that has been reported in the literature. While the exact mechanism is not fully understood, some of the factors thought to contribute to bone formation include the presence of an intact periosteum, age of the patient, infection, and immobility [12].

The presence of an intact periosteum is perhaps one of the most important factors contributing to osteogenesis. The periosteum is a layer of dense connective tissue that is attached to the adjacent bone via stable collagenous fibers known as Sharpey fibers. It consists of 2 layers: an outer fibrous layer and an inner cambium layer. The outer layer consists of fibroblasts and a vascular network. The inner layer contains osteoblasts, which play a crucial role in stimulating osteogenesis. When the periosteum is stripped from the bone, it is this layer, along with its osteogenic cells, that can provide a scaffold to promote spontaneous bone regeneration and healing [13].

The age of the patient is another important factor that contributes to mandibular bone regeneration. The majority of reported cases have been in young patients. The younger the patient, the more dense and vascular the periosteum. Increased vascularity brings the potential of improved osteogenesis. Incidentally, infection is also thought to jumpstart osteoblasts into triggering osteogenesis [14]. This corresponds to what took place in our patient, who was found to have an underlying infection in the cyst. Finally, immobilization has been predicted to contribute to bone formation [15]. A posterior edge of bone did remain present along the ramus, and would have provided the appropriate stabilization necessary for osteogenesis to occur.

Our patient underwent enucleation in the setting of known plans for mandibular distraction for RS. Initial follow-up X-rays did demonstrate thinning of the lateral cortex of the ramus, but this improved with time. CT imaging performed nearly 18 months after cyst enucleation demonstrated good overall bone formation (Fig. 2B). More impressive was the ability of the bone to regenerate following distraction. This case demonstrates the ability of the mandible to successfully regenerate after extensive cyst enucleation and bone removal, most likely secondary to an intact periosteum. It emphasizes the capacity of the pediatric population to undergo enucleation and also suggests that clinicians can be relatively aggressive in the surgical management of DCs when necessary.

In conclusion, DCs are benign mandibular cysts infrequently seen in the younger population. When a unilocular radiolucency is seen on imaging, the differential diagnosis should be broad and include possible malignant etiologies. Enucleation is the preferred treatment and may be employed even in instances where future mandibular distraction may be needed, such as in the case of patients with RS.

\section{ORCID}

Shayda Mirhaidari https://orcid.org/0000-0002-9544-9796

Ananth Murthy https://orcid.org/0000-0001-8109-6144

\section{PATIENT CONSENT}

The patient provided written informed consent for the publication and the use of their images.

\section{REFERENCES}

1. El Gehani R, Krishnan B, Orafi H. The prevalence of inflammatory and developmental odontogenic cysts in a libyan population. Libyan J Med 2008;3:75-7.

2. Daley TD, Wysocki GP, Pringle GA. Relative incidence of odontogenic tumors and oral and jaw cysts in a Canadian population. Oral Surg Oral Med Oral Pathol 1994;77:27680.

3. Mohan KR, Natarajan B, Mani S, et al. An infected dentigerous cyst associated with an impacted permanent maxillary canine, inverted mesiodens and impacted supernumerary teeth.J Pharm Bioallied Sci 2013;5:S135-8.

4. Hu YH, Chang YL, Tsai A. Conservative treatment of dentigerous cyst associated with primary teeth. Oral Surg Oral Med Oral Pathol Oral Radiol Endod 2011;112:e5-7.

5. Ko KS, Dover DG, Jordan RC. Bilateral dentigerous cysts-report of an unusual case and review of the literature. J Can Dent Assoc 1999;65:49-51.

6. Benn A, Altini M. Dentigerous cysts of inflammatory origin: a clinicopathologic study. Oral Surg Oral Med Oral Pathol Oral Radiol Endod 1996;81:203-9.

7. Tachibana T, Shimizu M, Shioda S, et al. Clinical observation on the cysts of the jaws in childhood especially on the 
follicular cysts. Japanese J Oral Maxillofac Surg 1980;26: $337-44$.

8. Suresh R, Janardhanan M, Joseph AP, et al. A rare case of dentigerous cyst in a one year old child: the earliest known reported occurrence. Head Neck Pathol 2011;5:171-4.

9. American Dental Association. 2016 Eruption charts [Internet]. Chicago, IL: American Dental Association; c2016 [cited 2016 April 10]. Available from: http://www.mouthhealth.org/en/az-topics/e/eruption-charts.

10. Nuri R, Kordi MR, Moghaddasi M, et al. Effect of combination exercise training on metabolic syndrome parameters in postmenopausal women with breast cancer. J Cancer Res Ther 2012;8:238-42.

11. Carlson ER. Odontogenic cysts and tumors. In: Miloro M GG, Larsen PE, et al., editors. Peterson's principles of oral and maxillofacial surgery. Hamilton: BC Decker; 2004. p.575-95.

12. Ahmad O, Omami G. Self-regeneration of the mandible following hemimandibulectomy for ameloblastoma: a case report and review of literature. J Maxillofac Oral Surg 2015;14: 245-50.

13. McKibbin B. The biology of fracture healing in long bones. J Bone Joint Surg Br 1978;60:150-62.

14. Adekeye EO. Rapid bone regeneration subsequent to subtotal mandibulectomy: report of an unusual case. Oral Surg Oral Med Oral Pathol 1977;44:521-6.

15. Park HW, Kim HJ, Park BM. Spontaneous regeneration of the lateral malleolus after traumatic loss in a three-year-old boy: a case report with seven-year follow-up. J Bone Joint Surg Br 1997;79:66-7. 